ARTICLE

DOI: $10.1038 / s 41467-018-02886-w$

\title{
Nucleotide resolution mapping of influenza $A$ virus nucleoprotein-RNA interactions reveals RNA features required for replication
}

\author{
Graham D. Williams ${ }^{1,2}$, Dana Townsend ${ }^{2}$, Kristine M. Wylie ${ }^{3,4}$, Preston J. Kim ${ }^{5}$, Gaya K. Amarasinghe ${ }^{5,6}$, \\ Sebla B. Kutluay ${ }^{2} \&$ Adrianus C.M. Boon ${ }^{1,2,5}$
}

Influenza A virus nucleoprotein (NP) association with viral RNA (VRNA) is essential for packaging, but the pattern of NP binding to VRNA is unclear. Here we applied photoactivatable ribonucleoside enhanced cross-linking and immunoprecipitation (PAR-CLIP) to assess the native-state of NP-vRNA interactions in infected human cells. NP binds short fragments of RNA ( 12 nucleotides) non-uniformly and without apparent sequence specificity. Moreover, NP binding is reduced at specific locations within the viral genome, including regions previously identified as required for viral genome segment packaging. Synonymous mutations designed to alter the predicted RNA structures in these low-NP-binding regions impact genome packaging and result in virus attenuation, whereas control mutations or mutagenesis of NP-bound regions have no effect. Finally, we demonstrate that the sequence conservation of low-NP-binding regions is required in multiple genome segments for propagation of diverse mammalian and avian IAV in host cells.

\footnotetext{
${ }^{1}$ Department of Medicine at Washington University School of Medicine, St Louis, MO 63110, USA. ${ }^{2}$ Department of Molecular Microbiology at Washington University School of Medicine, St Louis, MO 63110, USA. ${ }^{3}$ Department of Pediatrics at Washington University School of Medicine, St Louis, MO 63110, USA. ${ }^{4}$ The McDonnell Genome Institute at Washington University School of Medicine, St Louis, MO 63110, USA. ${ }^{5}$ Department of Pathology and Immunology at Washington University School of Medicine, St Louis, MO 63110, USA. ${ }^{6}$ Department of Biochemistry and Biophysics at Washington University School of Medicine, St Louis, MO 63110, USA. Correspondence and requests for materials should be addressed to A.C.M.B. (email: jboon@wustl.edu)
} 
nfluenza A virus (IAV) possesses a segmented, negative-sense RNA genome that is bound by the viral nucleoprotein (NP) throughout replication. Recent cryo-electron microscopy studies of the IAV ribonucleoprotein complex (vRNP) provide evidence for a NP-RNA complex structure with a corkscrew-like morphology and the tripartite polymerase complex at one end and a loop at the other end. However, current models conflict with each other and yield no information about RNA conformation, binding, or NP-RNA association ${ }^{1,2}$. NP is thought to coat viral RNA (vRNA) uniformly in cells and virus particles; however. uniform coating likely would preclude the possibility for RNA structure formation in RNPs. Numerous virus families utilize structured RNA elements for specific biological processes throughout infection, including genome packaging 3,4 . For example, viral RNA elements are required for efficient replication, mRNA splicing, and genome packaging of $\mathrm{IAV}^{5-7}$. Structure formation has been demonstrated with in vitro folded IAV vRNA and engineered genome segments, but the structural constraints imposed by nucleoprotein on vRNA generated during infection is not known ${ }^{8,9}$. Elucidation of the physiological interaction between NP and viral genomic RNA may provide novel insights into how IAV is capable of coordinating its lifecycle. Thus, we set out to determine the in vivo landscape of NP-vRNA interactions.

Infection and complete replication of IAV requires delivery of all eight genome segments into a recipient cell. All IAV segments require packaging signals derived from the termini on each segment ${ }^{10,11}$. Interaction between vRNAs has been demonstrated in vitro and disruption of packaging signals or interacting segment regions attenuated virus replication at the stage of genome packaging ${ }^{12-14}$. In many cases, mutation of a single segment leads to a significant decrease in the packaging efficiency of other segments ${ }^{5,15}$. Additionally, viral particles largely package only one copy of each genome segment ${ }^{16-18}$. Together, these results suggest that genome segments function as a multipartite, cooperatively packaged entity, possibly potentiated by segment-segment interactions, rather than a stochastically generated particle ${ }^{19,20}$.

In this study, we set out to determine how IAV NP interacts with vRNA during infection in cells. We show that the NP of IAV binds the vRNA non-uniformly and that regions of low-NP binding are enriched for predicted RNA secondary structures. Synonymous mutations designed to destabilize the predicted RNA structure attenuate IAV replication, whereas synonymous mutations that maintain the predicted RNA structure or mutations in NP-bound RNA regions have no effect on virus replication in vitro or in vivo. Viral attenuation is associated with an increase in defective virus production, suggesting that low-NPbinding regions and the predicted RNA structures are required for viral genome packaging.

\section{Results}

Nucleotide resolution mapping of NP-vRNA interactions. Photoactivatable ribonucleoside enhanced cross-linking and immunoprecipitation (PAR-CLIP) coupled to next-generation sequencing was used to resolve the interaction between the negative-sense RNA genome of IAV and NP during infection of human $293 \mathrm{~T}$ cells ${ }^{21}$. We infected human cells with WT-PR8 virus for $16 \mathrm{~h}$ in the presence of 4 -thiouridine (4-SU) to enhance crosslinking of NP-RNA complexes and then generated Illumina $1 \times 50$ sequencing libraries of the NP-bound RNA (Fig. 1a). The impact of 4-SU on viral replication was assessed in 293T cells. WT-PR8 replicated to equivalent titers 12,18 , and $24 \mathrm{~h}$ postinfection (hpi) in mock- or 4-SU-treated $(100 \mu \mathrm{M})$ cells (Fig. 1b). Additionally, NP localization after 4-SU treatment was assessed by confocal microscopy at $16 \mathrm{hpi}$, and no alteration was observed at this time point (Fig. 1b). These results suggest that 4-SU treatment does not substantially impact IAV nucleoprotein production or replication in human cells.

To determine the sensitivity and specificity of the PAR-CLIP assay, we performed Western blotting analysis for IAV NP and cellular $B$-actin on input lysate and immunoprecipitated proteins (Fig. 1c, bottom). Compared to immunoprecipitations performed without antibody or a control anti-HA antibody, immunoprecipitation with a monoclonal antibody (MAb) against IAV NP produced a specific band. UV-exposure of infected cells in the presence of 4-SU enabled greater recovery of NP-RNA complexes (Fig. 1c, top). The protein purity in the immunoprecipitate was verified by silver stain or Western blotting with an anti-IAV polyclonal serum (Supplementary Fig. 1a, b).

Influenza A nucleoprotein binds viral RNA non-uniformly. PAR-CLIP identified both human and virus-derived RNAs that interacted with NP. The procedure enriched for IAV RNA sequences relative to RNA-seq libraries (Fig. 1d), and the majority of the viral RNA sequences were derived from the negative-strand vRNA (Supplementary Fig. 2). The average length of the NPbound vRNA was 12 nucleotides (range 11-14, Fig. 1e).

We then compared PAR-CLIP and RNA-seq libraries to identify contiguous regions of vRNA that are significantly underand overrepresented among the NP-bound RNAs. Using the criteria of $>3$-fold difference, $Q<0.01$, and $\geq 18$ nucleotides long, we identified 24 regions in the viral genome that were low in NP binding relative to RNA-seq and 18 regions that met two of these three criteria that we did not investigate further (Supplementary Fig. 3, Supplementary Table 1). Moreover, four high-NP-binding regions where vRNA was overrepresented in PAR-CLIP libraries relative to the control RNA-seq sets were identified (Supplementary Table 1). The low-NP-binding regions together represent $\sim 10 \%$ of the viral genome and do not differ in base composition from the remainder of the genome (Fig. 1f). Analysis of the nucleotide sequences in low-NP-binding regions revealed that RNA secondary structures are predicted to form in the absence of NP binding in some of these regions (Supplementary Table 1$)^{5,7}$. Thus, NP binding might be affected by local secondary structures in the genome of IAV.

Segment 5 low-NP-binding regions are important for IAV. To assess the significance of the NP-bound or underrepresented vRNA regions, we selected six regions in segment 5 that have variable NP-binding profiles (Fig. $2 \mathrm{a}$ and Table 1). The lack of alternative reading frames and splicing of segment 5 mRNA made this segment highly suitable for extensive characterization of the significance of the low-NP-binding regions on IAV replication. Of the six regions, two were underrepresented (low-NP binding) in PAR-CLIP data sets $\left(\mathrm{NP}_{22-68}\right.$ and $\left.\mathrm{NP}_{1410-1495}\right)$. The $\mathrm{NP}_{1410-1495}$ region contains a previously hypothesized vRNA pseudoknot ${ }^{5}$. Additional regions of NP intermediate binding $\left(\mathrm{NP}_{145-175}, \mathrm{NP}_{456-490}, \mathrm{NP}_{584-608}\right.$, and $\left.\mathrm{NP}_{1058-1081}\right)$ were also included in the following studies. Computational prediction of RNA structures using RNAfold in low-NP-binding regions guided further mutational analysis, and these regions were mutagenized to either disrupt $\left(\mathrm{NP}_{22-68: \mathrm{A}}\right.$ and $\left.\mathrm{NP}_{1410-1495}\right)$ or maintain $\left(\mathrm{NP}_{22-68: \mathrm{B}}\right)$ the predicted secondary and tertiary structure formation (Supplementary Fig. 4). Mutant viruses bearing 2-7 synonymous structural nucleotide changes in these regions were generated and assessed for ability to replicate in vitro and in vivo.

WT and mutant viruses were subjected to a focus-forming assay in MDCK cells to determine replication competence in vitro (Fig. 2b). Mutations disrupting the segment 5 vRNA pseudoknot $\left(\mathrm{NP}_{1410-1495}\right)$ formation and destabilizing a predicted stem-loop 
structure $\left(\mathrm{NP}_{22-68: \mathrm{A}}\right)$ in the $3^{\prime}$ region of the vRNA segment resulted in reduction of focus area, as a measure of multi-cycle replication and spread (Fig. 2b). Conversely, mutation of intermediate NP-bound vRNA regions did not alter the focus area. Synonymous mutations in $\mathrm{NP}_{22-68}$ designed to maintain the predicted secondary structure $\left(\mathrm{NP}_{22-68: \mathrm{B}}\right)$, also did not affect focus size (Fig. 2b). Multi-cycle replication assays in MDCK cells of select mutant viruses confirmed these results (Fig. 2c). Finally, mice were inoculated with $10^{3} \mathrm{TCID}_{50}$ of each virus, and the presence of infectious virus in the lung was assessed $48 \mathrm{~h}$ later. WT-PR8 replicated to high titers at this time point whereas destabilizing mutations in low-NP-binding regions resulted in attenuation (Fig. 2d). Synonymous structural mutations in NPbound regions had no effect on virus infection in vivo (Fig. $2 \mathrm{~d}$ ).
These results collectively suggest that structural features of the low-NP-binding regions are important for IAV replication.

To determine the cause of attenuated replication of low-NPbinding mutant viruses, we evaluated effects on specific stages in the IAV lifecycle. All viruses displayed similar cytoplasmic distribution of NP $8 \mathrm{hpi}$ in MDCK cells when assessed by indirect immunofluorescence and confocal microscopy (Fig. 3a). The ability of the NP proteins from mutant viruses to facilitate the transcription and replication of a firefly luciferase reporter genome segment by the tripartite IAV polymerase complex was tested in human cells; all viruses displayed equivalent reporter activity (Fig. 3b). Similarly, infection of MDCK cells with all viruses generated equivalent amounts of viral antigen (NP) $8 \mathrm{hpi}$ when measured by flow cytometry (Fig. 3c). Therefore, the a

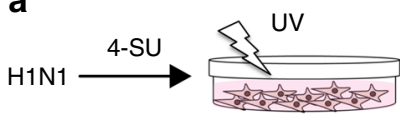

RNAse A digestion

NP

Immunoprecipitation

Purification of NP-RNA complexes

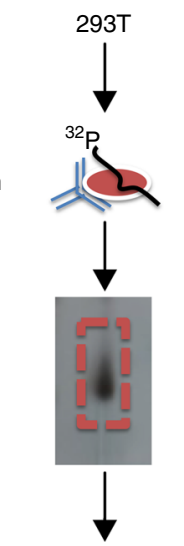

Illumina $1 \times 50$ NGS

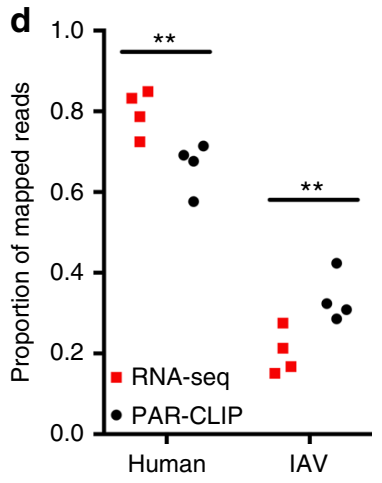

b
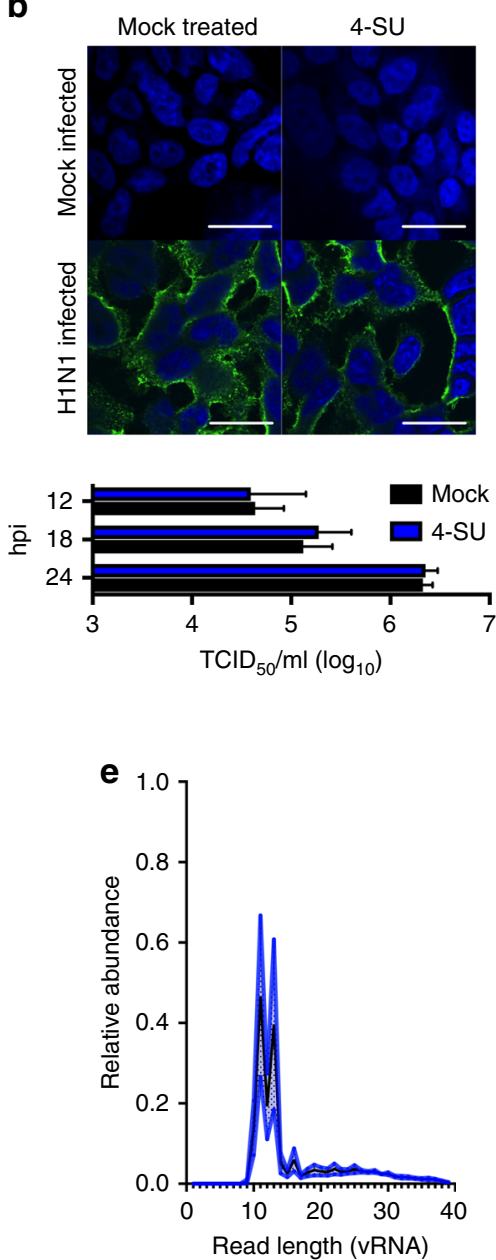

C
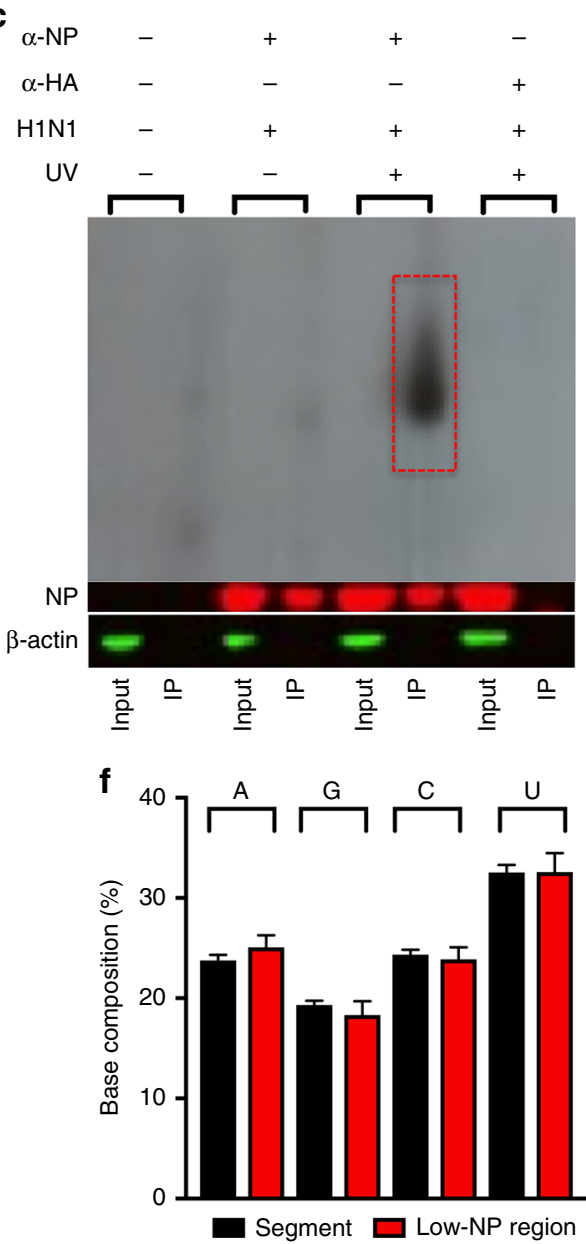

Fig. 1 Development of PAR-CLIP for IAV NP. a Schematic for IAV NP PAR-CLIP assay. $\mathbf{b}$ Effects of 4-SU on IAV replication. Viral replication (MOI = 0.1) in the presence or absence of 4-SU $(100 \mu \mathrm{M})$ was assessed by performing a growth curve at the indicated times in $293 \mathrm{~T}$ cells and titered by TCID ${ }_{50}$ assay in MDCK cells (bottom). Results are the average + s.e.m. of two experiments. NP localization was assessed following treatment and infection of $293 \mathrm{~T}$ cells by confocal microscopy (top). Immunofluorescence staining for NP (green) was assessed in the presence or absence of 4-SU and counterstained with DAPI to identify cellular nuclei (blue). Scale bar indicates $25 \mu \mathrm{m}$. c PAR-CLIP was conducted on 293T cells infected with WT-PR8 in the absence (lanes 1 and 2) or presence of a monoclonal antibody against to IAV NP (lanes 3-6) or viral hemagglutinin protein (HA) (lanes 7-8). The effect of UV cross-linking on binding of RNA to the viral NP is shown in lanes 3-4 (no UV) and lanes 5-6 (with UV). Radioactivity $\left({ }^{32} \mathrm{P}\right.$ ) is visualized by autoradiograph and the presence or absence of NP and cellular B-actin was done by western blot. The input sample and eluate are loaded in the uneven and even lanes, respectively. The results are representative of four independent experiments. Original western blots and autoradiograph are shown in Supplementary Fig. 7. d Proportion of PAR-CLIP or RNA-seq derived reads mapping to human or IAV genomes ( ${ }^{\star *} P<0.01$ by one-way ANOVA with multiple comparisons correction

(Kruskal-Wallis test), $n=4$ ). e Length of negative-sense viral RNA (vRNA) aligning reads was determined using FastX Toolkit and the number of reads of a certain length is plotted as a proportion of total vRNA mapping reads. Mean (black line) \pm s.e.m. (blue shading) of 4 experiments. $\mathbf{f}$ Nucleotide composition of low-NP-binding regions and IAV genome (displayed as average + s.e.m. base composition of all eight gene-segments). No significant differences were detected between groups (one-way ANOVA with multiple comparisons correction (Kruskal-Wallis test)) 


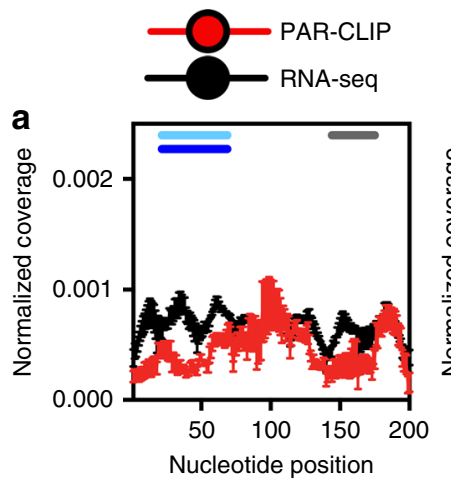

b

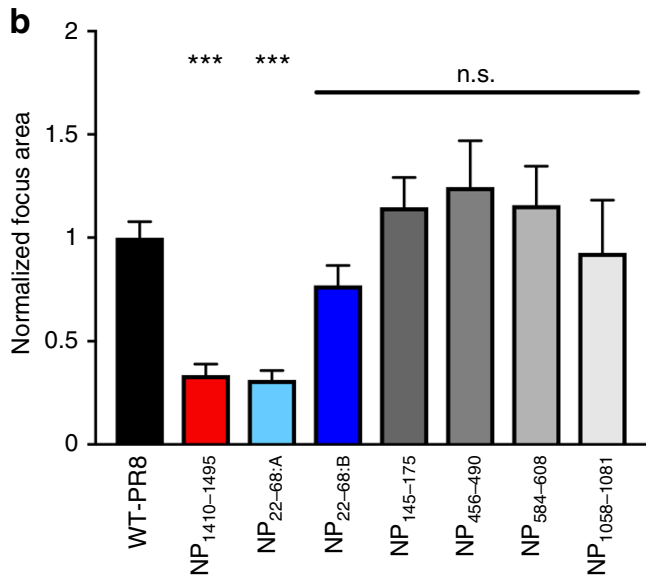

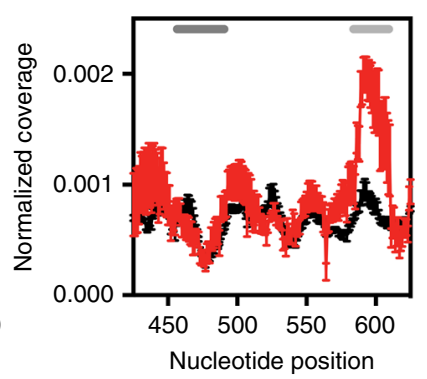
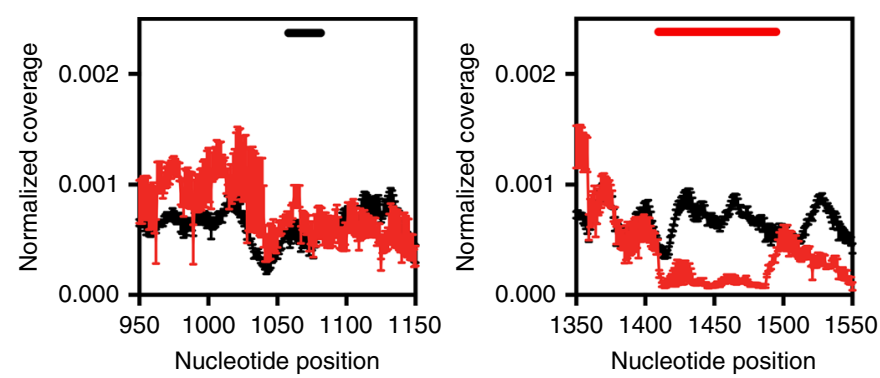

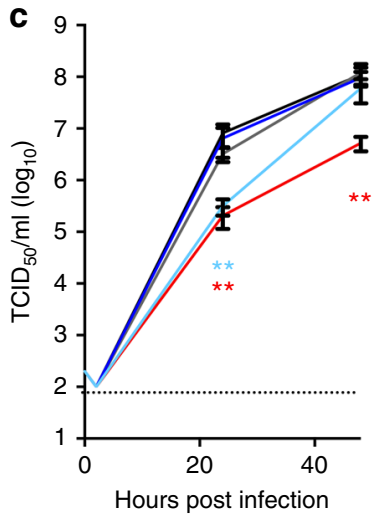

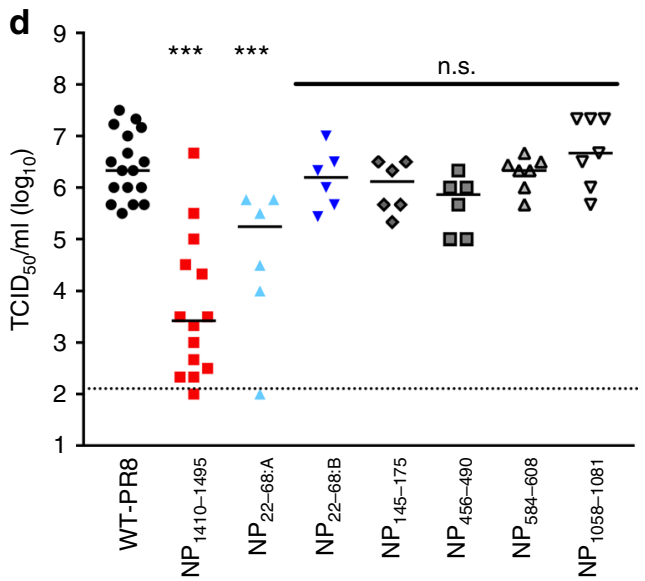

Fig. 2 Manipulation of vRNA in low-NP-binding regions that disrupts predicted RNA structure attenuates virus replication. a Normalized coverage \pm s.e.m. was determined for each nucleotide in the PAR-CLIP (red symbols) and RNA-seq (black symbols) libraries ( $n=4$ each). Six regions of interest (ROI) in segment 5 (NP) are highlighted, including two low-NP-binding regions $\left(\mathrm{NP}_{1410-1495}\right.$ and $\left.\mathrm{NP}_{22-68}\right)$ and four intermediate $\mathrm{NP}$-binding regions $\left(\mathrm{NP}_{145-175}\right.$, $\mathrm{NP}_{456-490}, \mathrm{NP}_{584-608}$, and $\mathrm{NP}_{1058-1081}$ ). Each ROI is indicated with a colored bar. $\mathbf{b}$ Focus area of WT-PR8 and NP mutant IAV. MDCK cells were infected with serial dilutions of the indicated viruses and overlayed with infection media (MO.1B) containing 1\% agarose and TPCK-trypsin. Seventy-two hours later cells were fixed, permeabilized, and stained for viral antigen (NP). Focus diameter was determined and normalized to WT-PR8 per experiment. Results are the average + s.e.m. of 3-5 experiments per virus (>60 foci each). c MDCK cells were infected with $\mathrm{MOI}=0.001$ of the indicated viruses and culture supernatant was collected at indicated time points then titered on MDCK cells. Results are the average $\pm \mathrm{s}$ e.m. $\mathrm{TCID}_{50} \mathrm{ml}^{-1}$ of $\mathrm{two} \mathrm{experiments}$ performed in duplicate. $\mathbf{d}$ C57BL/6 J mice were inoculated with $10^{3} \mathrm{TCID}_{50}$ of the indicated viruses in $30 \mu \mathrm{L}$. Lungs were collected, homogenized, and titered on MDCK

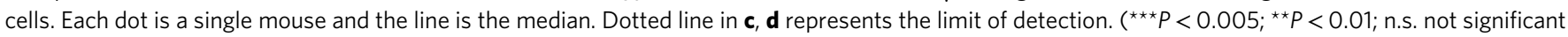
by one-way ANOVA with multiple comparisons correction (Kruskal-Wallis test))

synonymous mutations introduced within NP segment did not alter NP expression or its activity by all measurable outcomes.

We next assessed the virus particle to infectious unit ratio of WT and mutant PR8 viruses to evaluate potential defects in viral genome packaging. The infectious titer $\left(\mathrm{TCID}_{50} \mathrm{ml}^{-1}\right)$ of $\mathrm{NP}_{1410-1495}$ and $\mathrm{NP}_{22-68: \mathrm{A}}$ mutant viruses was significantly lower $(P<0.05)$ compared to all other viruses when normalized to HAunits, indicating a greater production of non-infectious particles (Fig. 3d). The ability of WT and mutant viruses to package all eight genome segments was assessed using a population level measure of relative vRNA segment abundance in purified viral particles $^{22}$. RNA from WT and mutant IAVs was subjected to RT-qPCR and the abundance of segments was normalized to segment 7 (Fig. 3e). Mutation of either the predicted pseudoknot $\left(\mathrm{NP}_{1410-1495}\right)$ or stem-loop structure $\left(\mathrm{NP}_{22-68: \mathrm{A}}\right)$ resulted in aberrant genome constellation stoichiometry. Consistent with the viral replication assays, mutation of intermediate NP-bound regions or mutations designed to maintain the predicted $3^{\prime}$ stemloop structure $\left(\mathrm{NP}_{22-68: \mathrm{B}}\right)$ result in unaltered ratios of genome segments in all but one segment-specific instance (Fig. $3 \mathrm{e}$ and Supplementary Fig. 5a). These results suggest that RNA structuredestabilizing mutations, in regions of vRNA poorly bound by NP, impact replication at the stage of coordinated genome packaging.
Finally, we determined the co-expression of NP and matrix (M) proteins in singly infected cells (Fig. 3f, multiplicity of infection (MOI) of 0.05$)^{23}$. In agreement with the RT-qPCR data, suggesting a packaging defect, a lower percentage of $\mathrm{NP}_{1410-1495}$ and $\mathrm{NP}_{22-68: \mathrm{A}}$ mutant virus-infected cells co-expressed both proteins compared to cells infected with WT or other mutant viruses. Notably, high MOI infection with multiple defective particles simultaneously infecting the same cell is able to overcome this defect (Supplementary Fig. 5b).

Additional low-NP-binding regions impact IAV replication. Low-NP-binding regions in segments 1,2 , and 8 also were evaluated for their contribution to viral replication by mutagenesis designed to disrupt predicted secondary and tertiary RNA structures (Fig. 4a-c, Supplementary Table 1 and Supplementary Data 1). Nucleotide substitutions that alter the predicted RNA secondary structure but have no impact on the protein, i.e., synonymous structural mutations, in segment 1 (PB2 1823-1944:A-C), segment $2\left(\mathrm{~PB}_{497-561: \mathrm{A}}\right)$ and segment 8 $\left(\mathrm{NS}_{23-86: \mathrm{A}}\right)$ reduced the focus area of mutant viruses in MDCK cells (Fig. 4d-f). Control mutations, designed to maintain the predicted vRNA structure in the low-NP-binding regions on segment 2 and $8\left(\mathrm{~PB}_{497-561: \mathrm{B}}\right.$, or $\left.\mathrm{NS}_{23-86: \mathrm{B}}\right)$, did not alter focus 
Table 1 Disruption of predicted RNA structures attenuates IAV

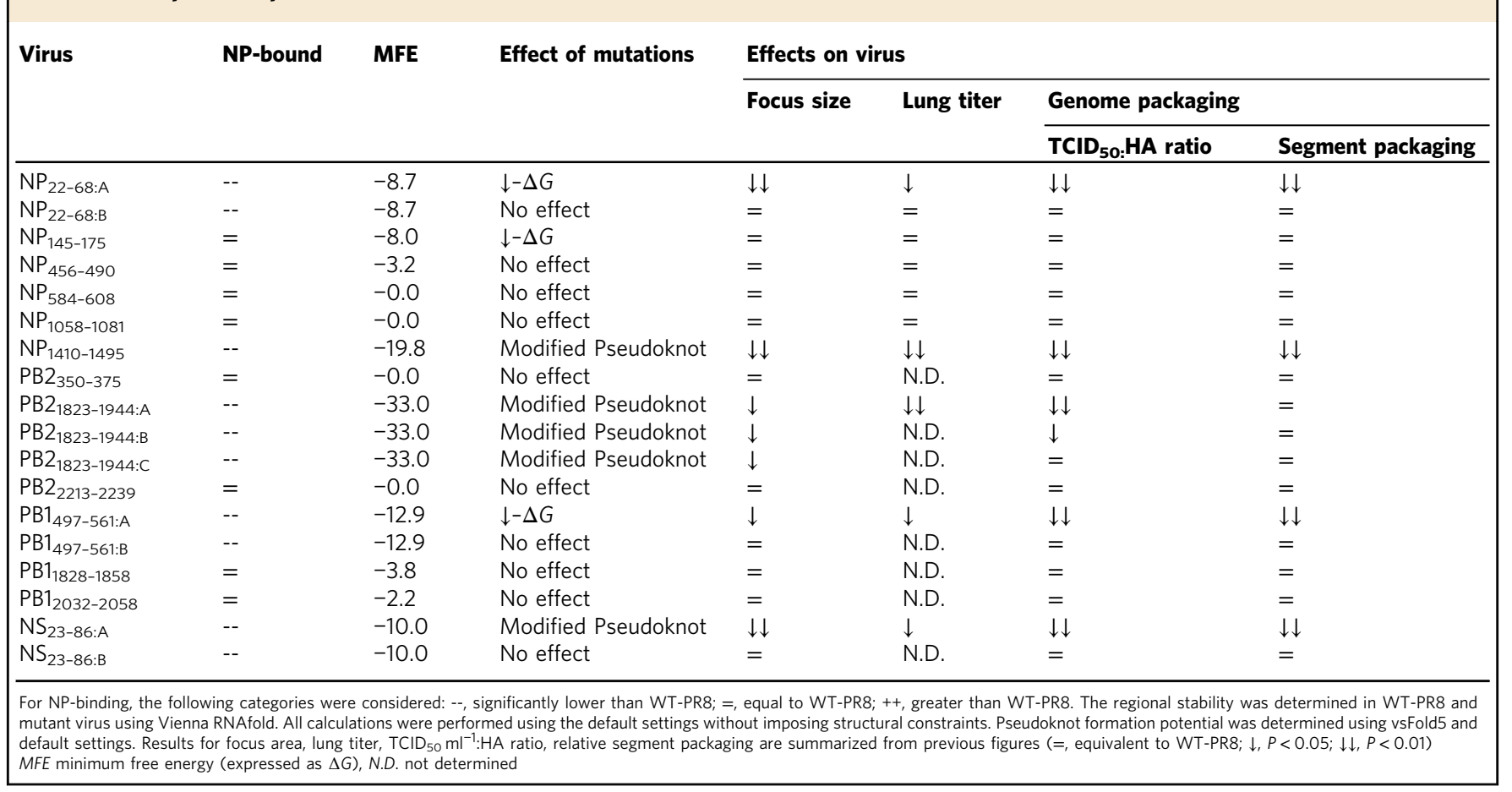

size (Fig. 4e, f). Similar to the attenuation of structurally disrupted segment 5 viruses, the $\mathrm{PB} 2_{1823-1944: \mathrm{A}-\mathrm{B}}, \mathrm{PB}_{497-561: \mathrm{A}}$ or $\mathrm{NS}_{23-86: \mathrm{A}}$ mutant viruses generated fewer $\mathrm{TCID}_{50}$ particles per HA-unit, while the control mutations $\left(\mathrm{PB1}_{497-561: \mathrm{B}}\right.$ or $\left.\mathrm{NS}_{23-86: \mathrm{B}}\right)$ did not (Fig. 4g-i). The $\mathrm{PB} 2_{1823-1944}$ mutant that showed the least attenuation in the focus-forming assay, $\mathrm{PB} 2_{1823-1944: C}$, had no discernable difference in TCID $_{50}$ per HA-unit. Segment 1 (PB2) and 2 (PB1) mutants did not display altered reporter activity (Supplementary Fig. 6a). In contrast to the impact of synonymous mutations in low-NP-binding regions, mutations in NP-bound regions in segment $1\left(\mathrm{~PB} 2_{350-375}\right.$ and $\left.\mathrm{PB} 2_{2213-2239}\right)$ and segment 2 (PB1 $1_{1828-1858}$ and $\mathrm{PB1}_{2032-2058}$ ) did not alter focus size or $\mathrm{TCID}_{50}$ per HA-unit (Fig. 4d, e, g, h, and Supplementary Fig. 5a). Specific manipulation of segment 8 vRNA sequence $\left(\mathrm{NS}_{23-86: \mathrm{A}}\right)$ resulted in decreased packaging of segments $3,4,5$, and 7 relative to segment 1 (Fig. 4j). Assessment of vRNA abundance in virus particles revealed that segment $2\left(\mathrm{~PB}_{497-561: \mathrm{A}}\right)$ mutant viruses packaged reduced levels of segment 6 vRNA (NA) (Fig. 4j). The PB2 1823-1944:A virus did not show altered segment abundance (Fig. $4 \mathrm{k}$ and Supplementary Fig. 6b). Finally, virus replication of the $\mathrm{PB} 2_{1823-1944: \mathrm{A}}, \mathrm{PB}_{497-561: \mathrm{A}}$ and $\mathrm{NS}_{23-86: \mathrm{A}}$ mutant viruses in mice was diminished 48 hpi compared to WT-PR8 (Fig. $4 \mathrm{k}$ ). Together, these data suggest that vRNA sequences that are low in NP binding help to coordinate packaging of a full complement of eight vRNA segments and changes to the predicted RNA structures in these regions results in virus attenuation. To demonstrate that synonymous structural mutations affect the stability of the predicted RNA structure, we performed RNA thermal stability assays using RNA oligomers corresponding to the low-NPbinding region $\mathrm{PB1}_{497-561}$. The thermal profile of the RNA containing the predicted structure-destabilizing mutations ( $\left.\mathrm{PB1}_{\text {497-561:A }}\right)$ was different from that of the WT RNA oligomer and the RNA containing synonymous mutations that maintained the RNA structure ( $\left.\mathrm{PB}_{497-561: \mathrm{B}}\right)$ (Supplementary Fig. 4d). Moreover, the thermal melt profile for the destabilizing mutations $\left(\mathrm{PB1}_{\text {497-561:A }}\right)$ indicated a lower thermal stability. In contrast, the thermal profile of the $\mathrm{PB1}_{497-561: \mathrm{B}} \mathrm{RNA}$ was similar to that of WT
RNA. Combined these data show that stable RNA structures are required for IAV replication and genome packaging.

Mutation of low-NP-binding regions attenuates diverse IAV. We evaluated the contribution of two low-NP-binding regions (PB2 1823-1944 and $\mathrm{NP}_{1410-1495}$ ), identified in WT-PR8, to the replication of a North American avian IAV (A/shorebird/Delaware/22/2006 (IAV-H7N3)) and the 2009 pandemic H1N1 IAV (A/California/04/2009 (IAV-pH1N1)). The PB2 and NP genesegment of IAV-PR8 and IAV-H7N3 are divergent and representative of mammalian and avian viruses, respectively (Fig. 5a, b, Supplementary Fig. 4). The NP gene-segment of IAV-pH1N1 is closely related to IAV-PR8 (Fig. 5a, Supplementary Fig. 4), while the PB2 gene-segment is more closely related to avian viruses (Fig. 5b, Supplementary Fig. 4). Isogenic IAV-H7N3 and IAV$\mathrm{pH} 1 \mathrm{~N} 1$ viruses, bearing synonymous mutations in the $\mathrm{NP}_{1410-1495}$ or $\mathrm{PB} 2_{1823-1944}$ regions, designed to disrupt the predicted local RNA structure, were evaluated for virus replication in vitro. Synonymous mutations designed to impact the predicted RNA secondary structure in these regions of IAV-H7N3 and IAV-pH1N1 attenuated viral replication, as measured by focus area (Fig. $5 c$, e). All mutant viruses displayed decreased TCID $_{50}$ per HA-unit (Fig. 5d, f). These results indicate that the RNA features, identified by PAR-CLIP in IAV-PR8, are important in genome packaging of diverse avian and human strains of IAV.

\section{Discussion}

We have identified the interaction landscape of IAV NP with viral RNA in the context of infected cells. Our findings indicate that binding of NP to viral RNA is restricted to an average of 12 nucleotides and the distance between two cross-linking sites is 25 nucleotides. These estimates agree with molecular models of NP-RNA interactions and the NP-binding footprint of a related orthomyxovirus ${ }^{1,20,25,26}$. Within this model, NP is excluded from consistent interaction with specific regions of vRNA and allows trans-interactions with either other genome segment vRNA or host and virus factors. The interaction between NP and the viral RNA was non-uniform and characterized by regions that were 
consistently low or high in NP binding. These results are in agreement with recent findings from Lee et al. ${ }^{27}$. About half of the low-NP regions are predicted to form secondary and tertiary RNA structures, based on computational analysis, and mutations designed to alter the stability of these predicted RNA structures resulted in attenuated virus infection (summarized in Table 1). The presence of RNA structures in RNP complexes may explain the pleomorphic nature of $\mathrm{RNPs}^{28}$. Several, mostly shorter lowNP-binding regions were not predicted to form stable structures.
These regions may represent a portion of a larger but less stable structure or have a different yet unknown function during IAV replication. At present, the function of high-NP-binding vRNA regions is unknown. These regions may have a role in intersegment NP-based interactions or be regions of high-NP density, enabled by position along the vRNP.

We examined NP-vRNA interactions at a late time point when a majority of viral RNA is distributed throughout the cytoplasm and thought to be within vRNP complexes ${ }^{19,29}$. In support of this,

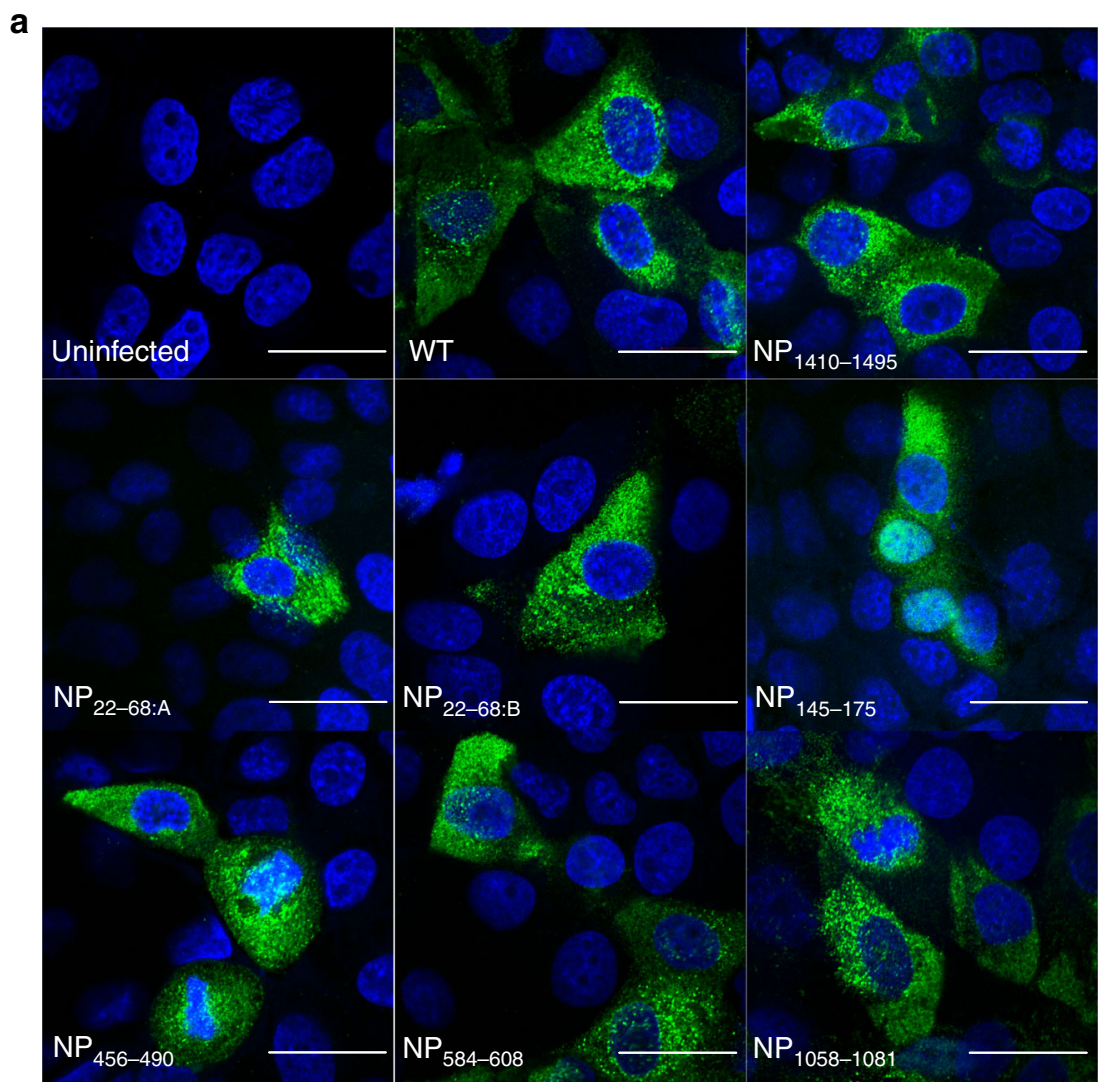

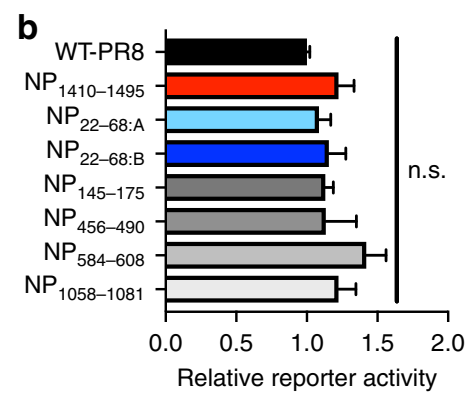

e

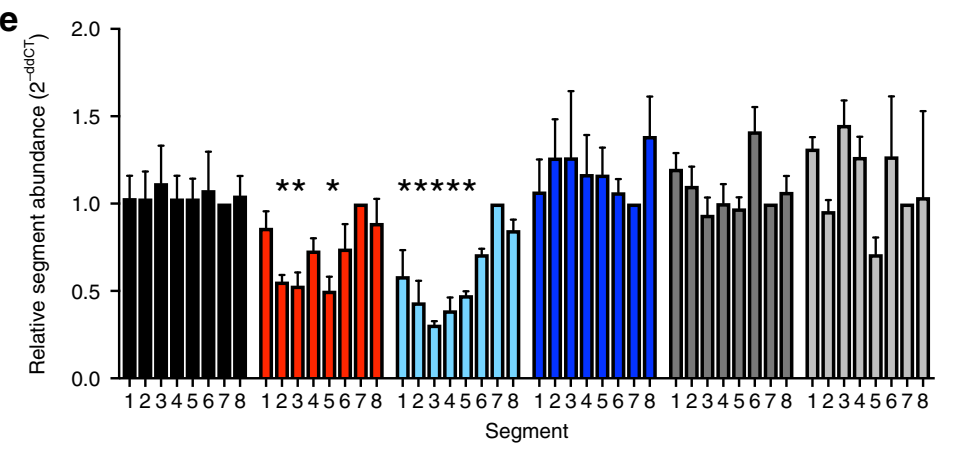

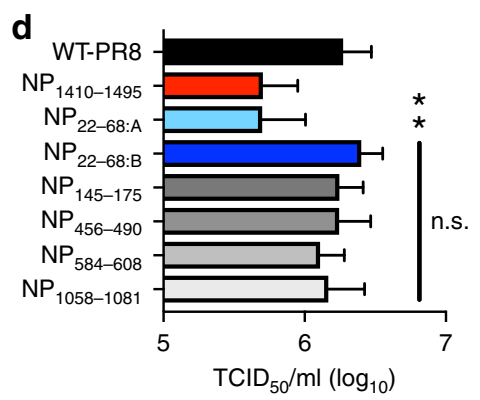

f

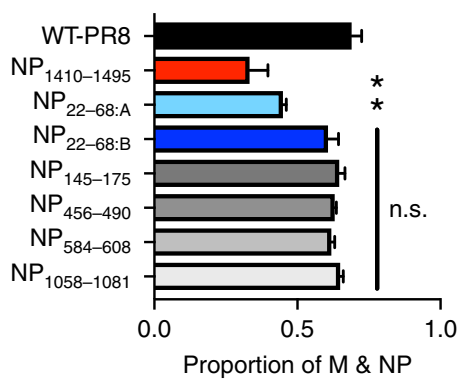

co-expression in infected cells 
the great majority of PAR-CLIP reads were obtained from negative-sense vRNAs.

We found many low-NP-binding RNA regions that overlap with previously predicted packaging and bundling signals near segment termini $\left(\mathrm{NP}_{22-68}, \mathrm{NP}_{1410-1495} \text {, and } \mathrm{NS}_{23-88}\right)^{5,30}$. However, additional low-NP-binding regions were identified throughout the segment body and outside of traditional packaging signals ${ }^{11,22,31}$. Our findings agree with studies assessing structure-guided mutagenesis of hypothetical vRNA structures (segment 7) or biochemical analyses of vRNA (segment 8) that largely overlap with low-NP-binding regions assessed here ${ }^{7,32}$. Additionally, we identified specific nucleotides required for copackaging of segment 2 (PB1) and segment 6 (NA), in a region recently implicated in directing reassortment outcomes and cosegregation of these segments in vitro ${ }^{33,34}$. Therefore, NP PARCLIP allowed us to directly identify potentially functional RNA regions in IAV genomes required for coordinated genome packaging.

As predicted RNA structures in some low-NP-binding regions are important for genome packaging, it suggests a requirement for inter-segment RNA interactions. Specific RNA interactions between genome segments have been demonstrated in vitro and ablation of these interactions lead to aberrant genome packaging; an outcome reversed by introduction of compensatory mutations in the interacting segment ${ }^{12}$. Many of the mutations that alter genome assembly also lead to an increase in number of defective viral particles ${ }^{30}$. The formation and nature of vRNA structures is likely contingent on the sequence composition and physical position of the nucleotides within a RNP complex. Additionally, manipulation of NP-bound regions, which may be unable to make inter-segment contacts due to NP-induced structural constraints on vRNA, did not impact virus replication. The presence of highly NP-bound vRNA regions may represent genomic regions that are spatially or structurally organized in a manner that allows improved protein binding, though we have not formally tested this hypothesis within this study.

IAVs can reassort and generate novel and potential pandemic strains. We tested if the regions identified in IAV-PR8 were required for replication of a divergent H7N3 virus. Synonymous changes designed to disrupt the predicted RNA structure in the same vRNA regions of two separate segments in both IAV-H7N3 and IAV-pH1N1 were attenuating and suggest conservations of these structural elements. Future examination of NP-binding of viral RNA in diverse strains of IAV is likely to identify overlapping and distinct high and low-NP-binding regions. These RNA features may be required for all viruses as coordinating packaging elements, but additional regions act as strain or lineage-specific packaging enhancers. Further, these strain or lineage-specific RNA features, required for genome packaging, may act as potential determinants of reassortment outcomes.
Prior experimental systems demonstrated that co-segregation of genome segments occurs during natural reassortment as well as lab-adaptation of virus strains to create new vaccines ${ }^{34,35}$. Finally, attenuation of genome packaging through silent, structural mutations has the potential to accelerate live attenuated vaccine production using native genome constellations to allow vaccination with all protein epitopes of a novel pandemic virus, without the need for master donor strains.

\section{Methods}

Cells. Madin-Darby Canine Kidney (MDCK) cells were maintained in minimal essential medium (MEM) with 5\% fetal bovine serum (FBS, Biowest), MEMvitamins (Gibco), L-glutamine (Gibco), and penicillin-streptomycin (Gibco). Human embryonic kidney cells (293-T) were maintained in Opti-MEM (Life Technologies) with $10 \%$ FBS, L-glutamine, and penicillin-streptomycin. MDCK and 293T cells were a kind gift from Dr. Richard Webby at St. Jude Children's Research Hospital.

Viruses. Eight bidirectional pHW2000 plasmids containing cDNA for A/Puerto Rico/08/1934 (H1N1), A/California/04/2009 (H1N1), or A/shorebird/Delaware/22/ 2006 (H7N3) were previously described ${ }^{35}$. Viruses were generated by transfection of all eight plasmids into co-cultures of $293 \mathrm{~T}$ and MDCK cells $(1 \mu \mathrm{g}$ per plasmid $)$ with polyethylenimine ( $8 \mu \mathrm{g}$ total). Wild-type (herein IAV-PR8 (WT-PR8), IAVpH1N1 (WT-pH1N1) or IAV-H7N3 (WT-H7N3) and mutant viruses were generated in the same manner with the exception of substituting individual plasmids harboring mutagenized DNA for the single indicated wild-type plasmid. The next day, the transfection mixture was removed and replaced with Opti-MEM containing MEM-vitamins, L-glutamine and penicillin-streptomycin. Forty-eight hours post-transfection, an additional $1 \mathrm{ml}$ of the same media containing $1 \mu \mathrm{g} \mathrm{ml}^{-1}$ TPCK-Trypsin (Worthington) was added to the co-culture. Seventy-two hours after addition of TPCK-Trypsin, culture supernatant was collected and clarified by centrifugation $(5 \mathrm{~min}, 350 \times \mathrm{g}$ ). Viral stocks were generated by infection of MDCK cells in a T75 flask. Cells were washed once with PBS, and $200 \mu$ of transfection supernatant was mixed with $25 \mathrm{ml}$ of infection media (M0.1B) composed of MEM containing vitamins, L-glutamine, penicillin-streptomycin, $0.1 \%$ Bovine Serum Albumin (Gibco) and $1 \mu \mathrm{g} \mathrm{ml}^{-1}$ TPCK-Trypsin for $48 \mathrm{~h}$. Stocks were aliquoted and stored at $-80^{\circ} \mathrm{C}$ until use, and all studies were conducted with passage 1 stocks following verification of mutant sequence identity. All viruses were generated at least twice independently. Sequences for primers utilized during mutagenesis of IAV plasmids are available in Supplementary Table 2.

IAV nucleoprotein PAR-CLIP and RNA-seq library generation. To identify interactions between viral RNA and IAV nucleoprotein, we adapted the protocol for PAR-CLIP ${ }^{21}$ coupled to next-generation sequencing to discern nucleotide resolution maps of protein-RNA interaction across the IAV genome. Confluent 293-T cells were infected at an MOI of 1 for $16 \mathrm{~h}$ in the presence of $100 \mu \mathrm{M} 4$ thiouridine (4-SU) and then cross-linked with ultraviolet light $(310 \mathrm{nM}, 500,000 \mu \mathrm{J}$ total energy, Boekel Scientific UV Crosslinker AH (115 V)). PAR-CLIP was performed essentially as described before ${ }^{21}$ with the exception of antibodies used to immunoprecipitate NP-RNA complexes: monoclonal antibodies HB65 (ATCC) or MAb8258 (EMD Millipore). On-bead Calf Intestinal Alkaline Phosphatase (CIP, NEB) treatment was used to dephosphorylate RNA ends followed by $5^{\prime}$ endlabeling with ${ }^{32} \mathrm{P}$ catalyzed by T4 Polynucleotide Kinase (PNK, NEB). Briefly, protein-RNA complexes were separated on 4-12\% SDS-PAGE gels and transferred to nitrocellulose membranes. ${ }^{32} \mathrm{P}$-labeled RNA was identified by autoradiography, excised, and extracted following proteinase K (New England Biolabs (NEB)) digestion. To account for potential bias introduced during adaptor ligation and sequencing, we also generated RNA-seq libraries using RNA extracted from

Fig. 3 Attenuating mutations in segment 5 impact coordinated genome packaging. a Confocal microscopy images depicting the localization of nucleoprotein (NP) $8 \mathrm{~h}$ post infection (hpi). MDCK cells were infected with the indicated virus (white text), and NP was identified using the MAb HB65 and Alexa-488-conjugated goat-anti-mouse secondary antibody by immunofluorescence. Cell nuclei are stained with DAPI. Panels depict the merged image of DAPI and NP staining. Fields are representative of two independent experiments. Scale bar represents $25 \mu \mathrm{m}$. $\mathbf{b}$ Dual-luciferase reporter assay to assess viral transcription and genome replication, each combination of plasmids was assessed 3-5 times with corresponding the WT-PR8 combination. (n.s., not significant by one-way ANOVA with multiple comparisons correction (Kruskal-Wallis)). c Mean fluorescence intensity (MFI) of NP in virally infected MDCK cells $(\mathrm{MOI}=0.05$, experiments were performed twice in duplicate) as revealed by flow cytometry. (n.s., not significant by one-way ANOVA with multiple comparisons correction (Kruskal-Wallis)). $\mathbf{d}$ Viral titer $\left(\mathrm{TCID}_{50} \mathrm{ml}^{-1}\right)$ of $4 \mathrm{HA}$-units of WT-PR8 and mutant viruses. Results are the average + s.e. $\mathrm{m}$. of three viral titrations and two HA assays per infectious virus titration. ( ${ }^{\star} P<0.05$; n.s., not significant by unpaired $t$ test). e Relative abundance of genome segments in purified WT or mutant viruses. All segments were compared to segment $7(M)$ VRNA and normalized to the average of WT-PR8 values using the $2^{-\mathrm{ddCt}}$ method ${ }^{22}$. Bars represent the mean of 3-6 independent virus preparations + s.e.m. $\left({ }^{\star} P<0.05\right.$; n.s., not significant by one-way ANOVA with multiple comparisons correction (Kruskal-Wallis)) f Proportion of infected cells co-expressing of matrix (M) and NP proteins in singly infected MDCK cells $(\mathrm{MOI}=0.05) 16 \mathrm{hpi}$ as revealed by flow cytometry. The average percentage of co-expression was calculated from two experiments performed in duplicate. (n.s., not significant by one-way ANOVA with multiple comparisons correction (Kruskal-Wallis)) 
uncross-linked cell lysates of influenza infected 293T cells from which RNA was isolated by TRIzol (Invitrogen) extraction, then $10 \mu \mathrm{g}$ RNA was fragmented by $\mathrm{Mg}^{+}$at $95^{\circ} \mathrm{C}$ for $12 \mathrm{~min}$ (NEB, Magnesium RNA Fragmentation Module). ${ }^{32} \mathrm{P}-$ labeling of this RNA was performed in solution with T4 PNK (NEB), and sizeselection of 10-100 nt fragments by Urea-Page (15\%) was performed. In both cases, total RNA was precipitated with ethanol, and used to prepare Illumina sequencing libraries. $3^{\prime}-\mathrm{OH}$ RNA previously prepared by CIP treatment was ligated to a $3^{\prime}$ adaptor using T4 RNA Ligase 2, truncated K227Q (NEB), according to manufacturer's instructions. The $5^{\prime}$ adaptor sequence contains NNN-degenerate nucleotides in addition to a sequence used for demultiplexing to facilitate collapsing of redundantly generated PCR products and ascertain the frequency of individual NP-RNA interaction events. This adaptor was ligated to the $5^{\prime}$-end of RNA using T4 RNA Ligase 1 (NEB). Following isolation of adaptor ligated and radiolabeled RNA, all subsequent library generation steps were identical. Independent PAR-CLIP or RNA-seq libraries were pooled and CDNA synthesized using a primer complementary to the $3^{\prime}$ adaptor (SuperScript III, Invitrogen). Libraries were amplified by PCR for 9-15 cycles using Phusion DNA polymerase (Phusion HF Mastermix, Thermo) and primers annealing to the $5^{\prime}$ and $3^{\prime}$ termini of the DNA that enable flow-cell binding. Size selection of libraries was conducted by extraction of amplicons from $6 \%$ urea gel electrophoresis, and then libraries were
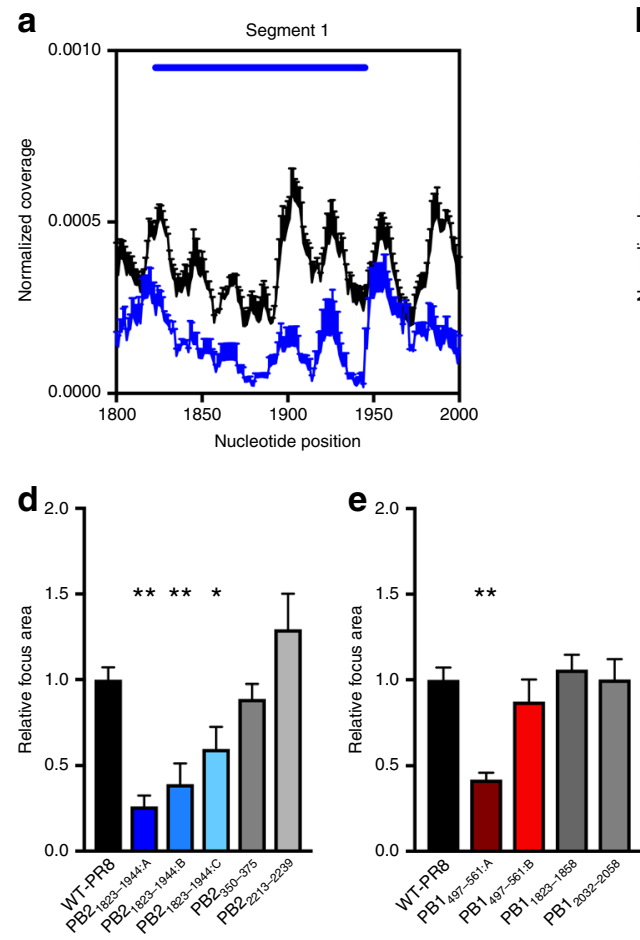

j

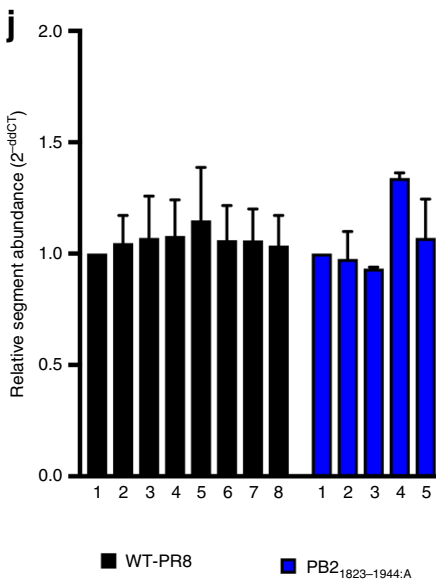

b
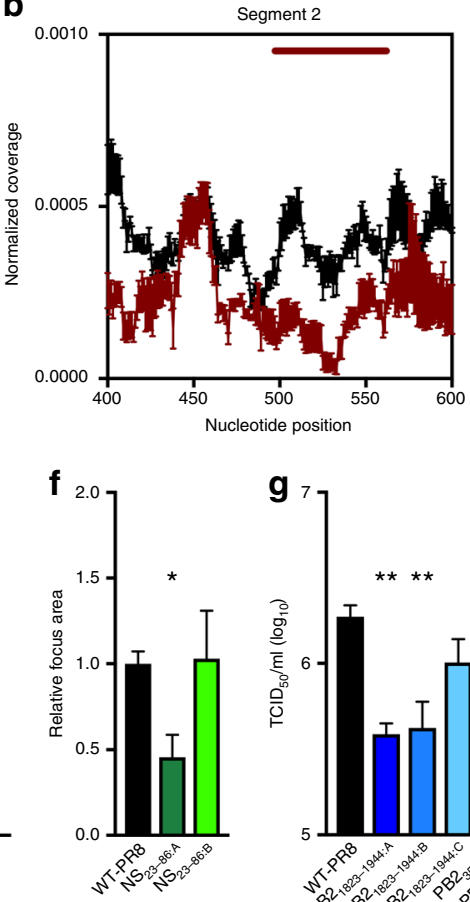
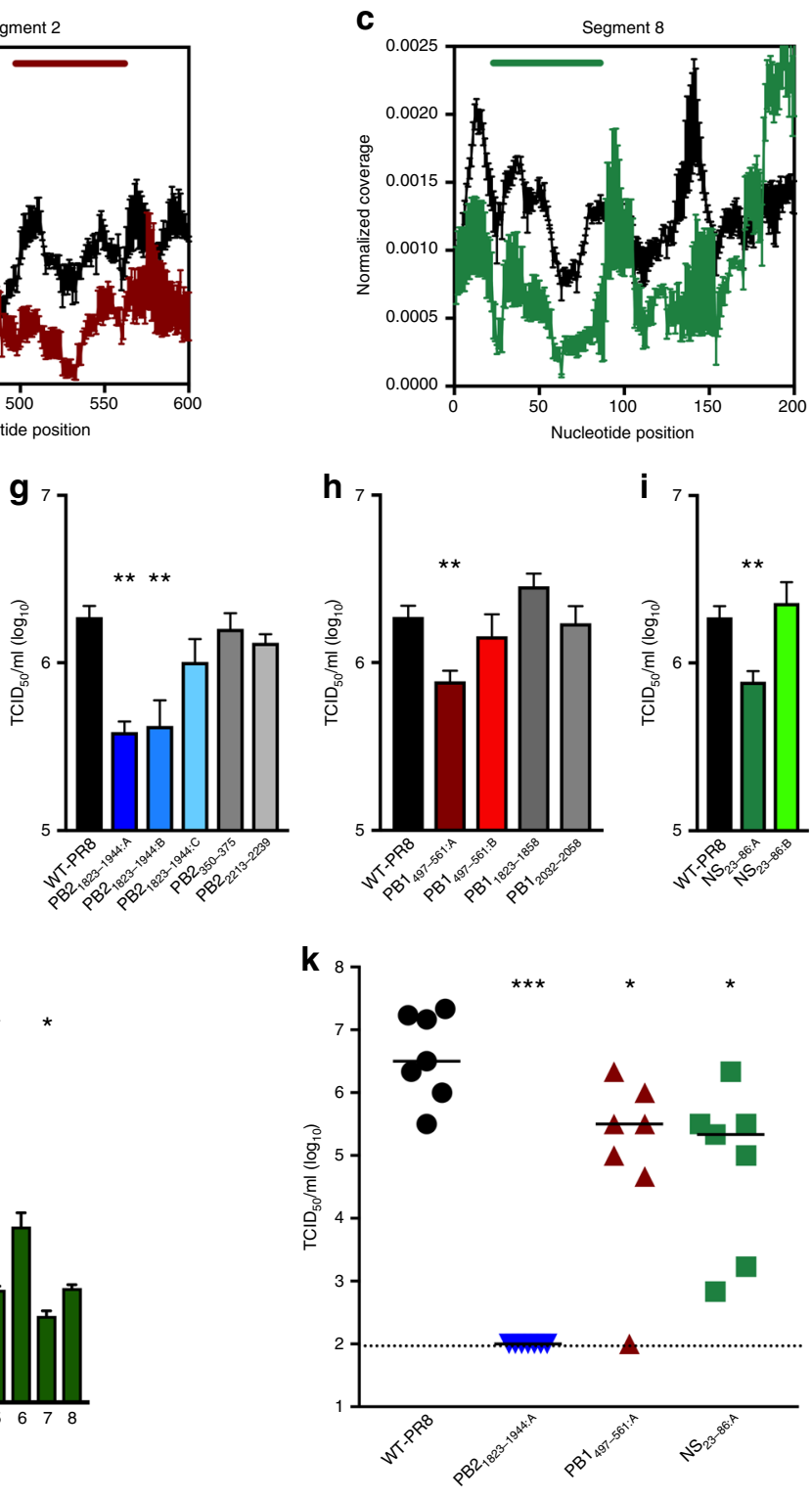

Fig. 4 Synonymous structural mutations in low-NP-binding regions in Segments 1, 2, and 8 attenuated virus replication and genome packaging. a-c Segment 1 (a, blue line), 2 (b, red line), and 8 (c, green line) low-NP-binding regions defined by PAR-CLIP analysis. Normalized coverage \pm s.e.m. was

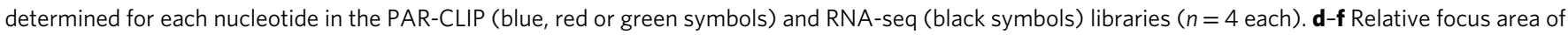
WT-PR8 and segment 1, 2, and 8 mutant viruses. Results are the average focus area + s.e.m. of 3 experiments per virus (>60 foci each). Statistical significance was determined by one-way ANOVA with a multiple comparisons correction (Kruskal-Wallis test). ${ }^{\star \star} P<0.01 ;{ }^{\star} P<0.05$. $\mathbf{g}-\mathbf{i} \vee$ iral titer

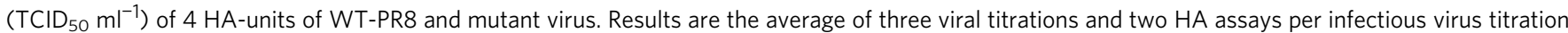
$\left(\mathrm{TCID}_{50}\right.$ assay and HA titration experiments, mean + s.e.m). Statistical significance was determined by unpaired $t$ test. ${ }^{\star \star} P<0.01$; ${ }^{\star} P<0.05$. $\mathbf{j}$ Relative abundance of genome segments in purified WT or mutant viruses. All segments were compared to segment 1 (PB2) vRNA and normalized to the average of WT-PR8 values using the $2^{-\mathrm{ddCt}}$ method $^{22}$. Bars represent the mean of 3-6 independent virus preparations + s.e.m. and statistical significance was determined by one-way ANOVA with a multiple comparisons correction (Kruskal-Wallis test). ${ }^{\star} P<0.05$. $\mathbf{k} \mathrm{C} 57 \mathrm{BL} / 6 \mathrm{~J}$ mice were inoculated with $10^{3}$ $\mathrm{TCID}_{50}$ in $30 \mu \mathrm{L}$. Lungs were collected $48 \mathrm{hpi}$, homogenized, and titered. Each dot is a single mouse and the line is the median. Dotted line in $\mathbf{k}$ represents the limit of detection and statistical significance was determined by one-way ANOVA with a multiple comparisons correction (Kruskal-Wallis test). $\left({ }^{\star \star \star} P<0.005,{ }^{\star} P<0.05\right)$ 
precipitated. Molarity of libraries was determined by qPCR (NEBNext, New England Biosciences) and size by Bioanalyzer (Agilent). Illumina sequencing on the HiSeq 2500 instrument $(1 \times 50$ bp reads) was performed by the Genome Tech nology Access Center at Washington University in St. Louis.
Next-generation sequencing. All NGS data were analyzed on the Washington University in Saint Louis School of Medicine's McDonnell Genome Institute (MGI) cluster using publically available analysis programs (FastX toolkit, Bowtie, and SAMtools) and in-house scripts ${ }^{21}$. PAR-CLIP and RNA-seq data were generated from four independent experiments. For PAR-CLIP and RNA-seq libraries we used an analysis pipeline that collapsed unique barcoded reads, removed adaptors, and a

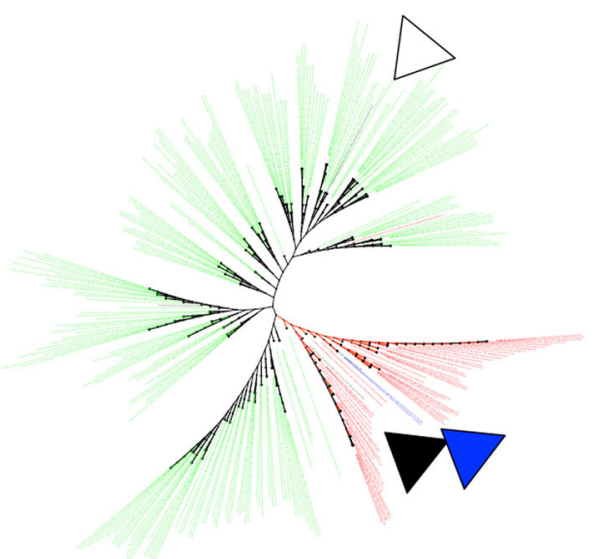

4.0 b

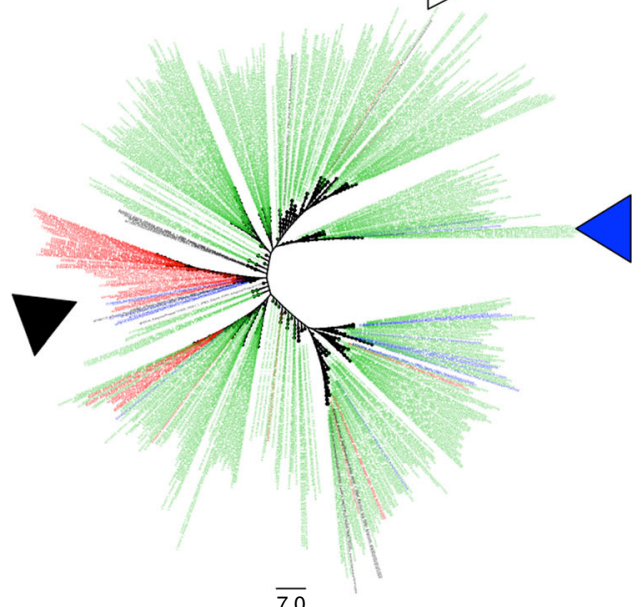

A/Puerto Rico/08/1934 (H1N1)

A/shorebird/Delaware/22/2006 (H7N3)

A/California/04/2009 (H1N1)
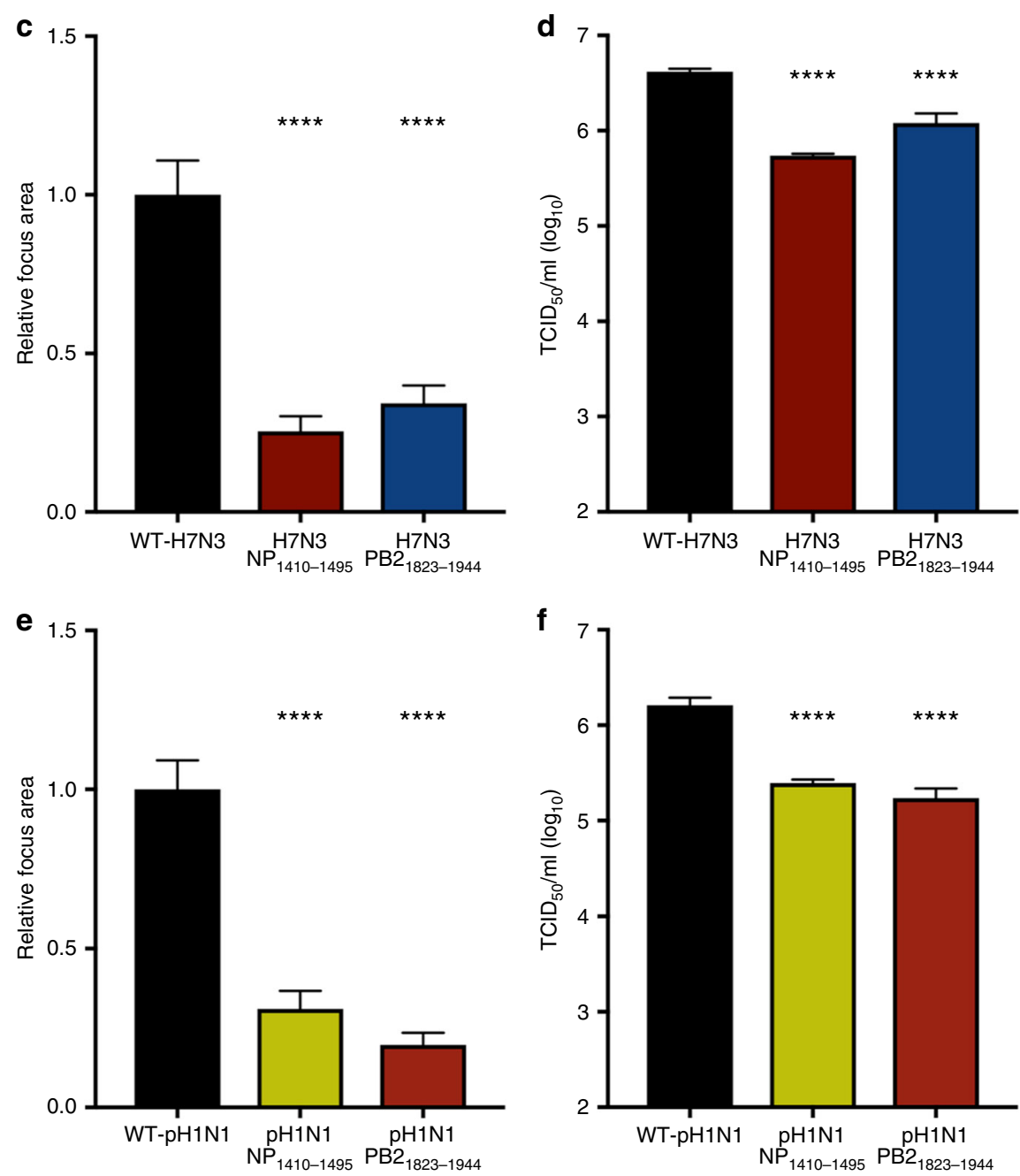
aligned them to the viral genome. Strandedness was determined post-alignment and all subsequent analysis was performed on negative-sense viral genomic RNA (vRNA). For reads that mapped to the viral RNAs, we normalized the number of reads per nucleotide to the total number of reads per genome segment to yield a normalized coverage ratio for both PAR-CLIP and RNA-seq libraries. We then compared the normalized coverage for each preparation at nucleotide resolution using an unpaired $t$ test with False Discovery Rate (FDR) correction

(Benjamini-Hochberg) ${ }^{36}$. vRNA nucleotides with a FDR minima $Q<0.01$ and fold-change $>3$ were identified and these regions were extended to the final nucleotide of each regionm, where $Q \leq 0.1$. These areas represent low-NP or high$\mathrm{NP}$-binding regions of interest (ROI). ROI $\geq 18 \mathrm{nt}$ were subjected to RNA structure analysis with mFold, RNAfold, and vsFold5 to determine computationally the theoretical minimal free energy $(\Delta G)$ of each region as well as the potential secondary or tertiary RNA structure formation ${ }^{37,38}$. Nucleotide composition of ROIs was determined by calculating the percent of $\mathrm{A}, \mathrm{G}, \mathrm{C}$, or $\mathrm{U}$ in each region and comparing them to the percent of each nucleotide in the IAV genome. PAR-CLIP data sets were compared to RNA-seq data sets at each nucleotide position on a persegment basis and positions using unpaired $t$ test with FDR correction as above with a threshold of $Q=0.05$. Sites of statistically enriched transition in PAR-CLIP data sets were tabulated and the sequence distance between these positions was calculated. Transition events with a step-size of 1, i.e. sequential nucleotides both with high transition rate, and were excluded from analysis because they likely represent single NP-RNA interactions of adjacent 4-SU nucleotides in vRNA. Transition rate for four PAR-CLIP libraries were averaged prior to distance calculation.

Generation of IAV containing altered RNA structures. Mutant selection was performed after analysis of open reading frames to assess potential nucleotide degeneracy while retaining coding sequence. Subsequently, we selected five underrepresented ROIs in four segments $(1,2,5$, and 8$)$ for functional assessment of manipulating predicted RNA structures on viral replication. We also identified four regions in segment 5 , amenable to extensive silent mutagenesis that were either highly bound or represented at the same frequency in PAR-CLIP and RNAseq data sets. We introduced between 2 and 7 synonymous mutations simultaneously in silico to the identified regions and reassessed structural stability or pseudoknot formation using the same structure prediction programs. We selected variant codon combinations that would disrupt or maintain the predicted vRNA structure but not change the encoded amino acid, alter codon usage, or disrupt alternative reading frames or splicing events. Once predicted destabilizing mutations were identified, mutant viruses were generated. Mutations were introduced into pHW2000 bidirectional plasmids by inverse PCR with primers (see Supplementary Table 2) including selected mutations and unique ligation sites. PCR products were gel extracted and digested with either BsmbI (New England Biolabs, (NEB)) or AarI (Thermo Fisher Scientific) restriction enzymes and DpnI (NEB) to remove residual parent plasmid. Digested PCR products were PCR purified and ligated using Instant Sticky End Ligase (NEB). Ligation products were transformed into E. coli, plated on selective LB Agar. Colonies were selected and grown overnight in LB broth and selective antibiotics. All plasmids were grown in $200 \mathrm{ml} \mathrm{LB}$ Broth prior to preparation by Qiagen HiSpeed Endotoxin-Free MaxiPrep and verified by Sanger Sequencing (Genewiz).

Influenza A virus focus-forming assay. Six-well plates of MDCK cells were plated and inoculated with $1 \mathrm{ml}$ of 10 -fold serially diluted virus stock for $1 \mathrm{~h}$ in M0.1B. After $1 \mathrm{~h}$, the inoculum was removed, and replaced with an overlay of $1 \%$ Low Melting Point Agarose in MEM supplemented with $1 \mu \mathrm{g} \mathrm{ml}^{-1}$ TPCK-Trypsin. Seventy-two hours post infection cells were fixed in 5\% formaldehyde, permeabilized with $0.1 \%$ saponin in HBSS, and stained for NP protein with $1 \mu \mathrm{g} \mathrm{ml}^{-1}$ biotinylated anti-NP (mAB8258b, Millipore). Foci were visualized by addition of HRP-conjugated streptavidin and TruBlue streptavidin substrate and imaged on a Biospot reader (Cellular Technology Limited). Individual foci were counted and area was calculated using the Analyze Particles extension within Fiji ${ }^{39}$. The area of the foci formed by mutant viruses was normalized to the average of WT-PR8 foci per experiment. A minimum of 60 foci was analyzed per virus.

Confocal microscopy. We infected $293 \mathrm{~T}$ cells in the presence or absence (mock) of 4-SU with WT-PR8 (MOI of 1) for $16 \mathrm{~h}$. Briefly, 293T cells were seeded on glass coverslips coated with poly-D lysine overnight. Cells were infected with WT-PR8 for $16 \mathrm{~h}$ then fixed with $4 \%$ methanol-free PFA in PBS (pH 7.4), washed with PBS, permeabilized with saponin, then NP staining was performed using anti-NP MAb HB65 $\left(1 \mu \mathrm{g} \mathrm{ml}^{-1}\right)$ and goat-anti-mouse Alexa-488 (Invitrogen). Nuclei were counterstained with DAPI. Coverslips were mounted to slides with ProLong ${ }^{\circledR}$ Diamond Antifade Mountant (Molecular Probes). To determine if viral protein production was impacted by synonymous mutations, we utilized confocal microscopy to visualize the distribution of NP during infection as a marker of viral protein production and a proxy for trafficking of vRNPs. MDCK cells were infected with WT-PR8 and indicated mutant viruses (MOI of 0.2) for $8 \mathrm{~h}$, then fixed and permeabilized as before. NP staining was performed as above using anti-IAV-NP MAb HB65 and Goat-anti-Mouse Alexa-488 (Invitrogen). Slides were imaged with Zeiss LSM 880 Confocal Laser Scanning Microscopy with Airyscan and analyzed with Zeiss Zen Black Software performed within the Molecular Microbiology Imaging Facility at Washington University in Saint Louis.

Influenza A virus reporter assay. To assess the impact of silent mutations on polymerase complex activity, pHW2000 plasmids encoding WT or mutant PB2, PB1, PA, and NP were utilized in a dual-luciferase reporter assay as previously described $^{35}$. A vRNA-like firefly luciferase reporter plasmid and a Renilla luciferase expression plasmid also were included. Briefly, 293T cells were seeded into 24-well plates and transfected with equal amounts of all six plasmids (500 ng DNA total) in Opti-MEM containing TransIT-LT1 (Mirus). Cells were maintained at $37^{\circ} \mathrm{C}$ for the duration of the experiment. Forty-eight hours later, cells were lysed for analysis of luciferase activities (Promega). Each combination of polymerase proteins (set of plasmids) was examined in duplicate. The relative light units (RLU) of firefly luciferase activity were normalized to the RLU for Renilla luciferase activity within the same sample to account for differences in transfection efficiency between wells and experiments. The activity of each plasmid set containing a mutant segment was normalized to the activity of WT-PR8.

Flow cytometry. To determine the amount of NP generated at a fixed time during infection, we infected $2 \times 10^{5}$ MDCK cells (MOI of 0.2 ). Eight hours post-infection, cells were collected and fixed. Intracellular staining of IAV antigen was performed as above using an anti-IAV NP primary antibody (HB65) $\left(1 \mu \mathrm{g} \mathrm{ml}^{-1}\right)$ and an Alexa-488-conjugate goat-anti-mouse secondary antibody (Invitrogen). The mean fluorescence intensity (MFI) of $\mathrm{NP}^{+}$cells was plotted and calculated from two experiments performed in duplicate. Co-expression of NP and M in MDCK cells was determined $16 \mathrm{hpi}$ with an MOI of 5 and 0.05 . Infected cells were fixed and stained as above with the additional staining step of utilizing mouse-anti-M primary antibody (M2-1C6) $\left(2 \mu \mathrm{g} \mathrm{ml}^{-1}\right)$ and goat-anti-mouse Alexa-647 secondary. A second intracellular staining step utilizing biotinylated mouse-anti-NP (mAb8258b) $\left(1 \mu \mathrm{g} \mathrm{ml}^{-1}\right)$ and Alexa-488 conjugated streptavidin was then performed. The frequency of co-expression was calculated by determining the number of cells expressing NP or M as well as those expressing both. The percentage of infected cells co-expressing NP and M was calculated by dividing co-expressing cells by all cells expressing one or more viral protein as previously described ${ }^{23}$. Analysis of viral proteins was determined using a flow cytometer and FlowJo software (Tree Star).

Multi-step replication of influenza A virus. MDCK cells $\left(2 \times 10^{5}\right)$ were seeded in 24-well plates and inoculated the next day with $200 \mathrm{TCID}_{50}$ of IAV. Cells were washed once with PBS before addition of inoculum in M0.1B and incubated for $1 \mathrm{~h}$ at $37^{\circ} \mathrm{C}$. Subsequently, the cells were washed twice with PBS and $1 \mathrm{ml}$ of M0.1B supplemented with $1 \mu \mathrm{g} \mathrm{ml}^{-1}$ TPCK-Trypsin was added to each well. Culture

Fig. 5 VRNA regions required for PR8 replication are required for replication of contemporary avian and human IAV. a Phylogenetic analysis of segment 5. WT-PR8, WT-H7N3, or WT-pH1N1 IAV are indicated by corresponding, labeled arrows. Phylogenies were created from randomly sampled full-length segment 5 sequences downloaded from NCBI IVR. Alignment performed in MEGA (version 7) using MUSCLE. Phylogenetic trees created using the Maximum Parsimony method included in MEGA. Phylogenies were visualized in FigTree and manually annotated. Green shading represents segment sequences derived from avian viruses; red represents human viruses; and blue represents swine viruses. b Phylogenetic analysis of segment 2 performed as in a. c Relative focus area of WT-H7N3 and mutant viruses + s.e.m. The results are the average of 3 independent experiments and $>60$ foci per virus. Statistical significance was determined by one-way ANOVA with multiple comparisons correction (Kruskal-Wallis test). ${ }^{\star \star \star \star} P<0.001$. $\mathbf{d}$ Viral titer $\left(\mathrm{TCID}_{50} \mathrm{ml}^{-1}\right)$ of $4 \mathrm{HA}$-units of WT-H7N3 and mutant virus. Results are the average of three viral titrations and two HA assays per infectious virus titration $\left(\mathrm{TCID}_{50}\right.$ assay and $\mathrm{HA}$ titration experiments, mean + s.e.m.). Statistical significance was determined by unpaired $t$ test. ${ }^{\star \star \star \star} P<0.001$. e Relative focus area of WT-pH1N1 and mutant viruses + s.e.m. The results are the average of 3 independent experiments and $>60$ foci per virus. Statistical significance was determined by one-way ANOVA with multiple comparison corrections (Kruskal-Wallis test). ${ }^{\star \star \star \star} P<0.001$. $\mathbf{f}$ Viral titer $\left(T_{C} \mathrm{CID}_{50} \mathrm{ml}^{-1}\right.$ ) of $4 \mathrm{HA}-\mathrm{units}$ of WT and mutant virus. Results are the average of three viral titrations and two HA assays per infectious virus titration $\left(T_{C I D_{50}}\right.$ assay and $H A$ titration experiments, mean + s.e.m.). Statistical significance was determined by unpaired $t$ test. ${ }^{\star \star \star \star} P<0.001$ 
supernatants were collected at 24 and 48 hpi and the amount of infectious virus was quantified by titration on MDCK cells.

Titration of influenza A virus (TCID ${ }_{50}$ ). Confluent monolayers of MDCK cells were grown overnight in 96-well tissue culture plates. The next day, the cells were washed with PBS and inoculated with 10 -fold serial dilutions of culture supernatant or lung homogenate for $1 \mathrm{~h}$ in $\mathrm{M} 0.1 \mathrm{~B}$ at $37^{\circ} \mathrm{C}$. After $1 \mathrm{~h}$, the inoculum was removed and replaced with M0.1B supplemented with $1 \mu \mathrm{g} \mathrm{ml}^{-1}$ TPCK-Trypsin and incubated for $72 \mathrm{~h}$. Presence of virus was determined by hemagglutination assay using $0.5 \%$ turkey red blood cells. $\mathrm{TCID}_{50}$ was determined by the Reed-Muench method ${ }^{40}$.

Infection of mice with influenza viruses. Male C57BL/6 J mice (5-6 weeks of age) were bred in-house in a barrier facility at Washington University School of Medicine, St. Louis, MO or purchased from Jackson Laboratories. Mice received food and water ad libitum and all experiments were conducted in accordance with rules of the Institutional Animal Care and Use Committee. Mice were anesthetized with isoflurane in an airflow chamber and then inoculated intranasally with $30 \mu \mathrm{L}$ of sterile PBS containing 1000 TCID $_{50}$ of WT-PR8 or the indicated mutant virus. Forty-eight hours post-infection, lungs were collected and homogenized in $1 \mathrm{ml}$ $\mathrm{M} 0.1 \mathrm{~B}$, cleared by centrifugation at $1200 \times \mathrm{g}$ for $5 \mathrm{~min}$, then stored in aliquots at $-80^{\circ} \mathrm{C}$. Viral titers from lung homogenates were determined by $\mathrm{TCID}_{50}$ assay.

Segment abundance RT-qPCR. MDCK cell derived stocks of WT-PR8 or mutant viruses were clarified by centrifugation at $1200 \times g$ for $5 \mathrm{~min}$, passed through a $0.22 \mu \mathrm{M}$ filter, and pelleted on $30 \%$ sucrose cushion by ultracentrifugation (Beckman SW32ti swinging bucket rotor, $27 \mathrm{~K} \mathrm{RPM}, 4^{\circ} \mathrm{C}$ for $90 \mathrm{~min}$ ). Pelleted virus particles were directly resuspended in $350 \mu \mathrm{l}$ TRK lysis buffer, and RNA purified immediately and eluted in $30 \mu \mathrm{LEPC} \mathrm{H}_{2} \mathrm{O}$ (Total RNA Kit I, Omega). cDNA was synthesized from $5 \mu \mathrm{l}$ of RNA with SSIII Reverse transcriptase (Invitrogen) and a vRNA specific primer ${ }^{22}$. Total cDNA was diluted $1: 10,000$ and used to quantify each of the 8 genome segments by SYBR Green qPCR (PowerUp ${ }^{\text {TM }}$ SYBR $^{\circledR}$ Green Master Mix) with primer pairs previously published ${ }^{22}$. Relative abundance of each genome segment was calculated as before, except with normalization to Segment 1 or 7 depending on the virus assessed.

RNA thermal denaturation experiments. The RNA oligomers corresponding to $\mathrm{PB1}_{497-561}, \mathrm{PB1}_{497-561: \mathrm{A}}$, and $\mathrm{PB} 1_{497-561: \mathrm{B}}$ (Supplementary Table 2) were synthesized commercially (Integrated DNA Technologies). RNA was diluted in buffer $(100 \mathrm{mM} \mathrm{NaCl}$ and $50 \mathrm{mM} \mathrm{KCl})$ to a final concentration of $10 \mu \mathrm{M}$, incubated at $95^{\circ} \mathrm{C}$ for $5 \mathrm{~min}$ and flash cooled on ice to induce folding. Unfolding of RNA secondary structure was monitored by CD spectroscopy at 210 and $260 \mathrm{~nm}$ on a Chirascan CD spectrometer (Applied Photophysics) ${ }^{41}$. RNA was heated from 5 to $95^{\circ} \mathrm{C}$ at a rate of $1{ }^{\circ} \mathrm{C} / \mathrm{min}$ and $\mathrm{CD}$ readings collected every $2^{\circ} \mathrm{C}$. A linear, leastsquares program was used to fit the transition region of thermal melting curves. By definition, the melting temperature of nucleic acid is the temperature at which $50 \%$ of all nucleic acid becomes single-stranded for a single cooperative transition ${ }^{24}$. The ellipticity at which half of the RNA is denatured was calculated using the equation shown below and modified from (42):

$$
\mathrm{Fd}=(\theta \mathrm{n}-\theta \mathrm{obs}) /(\theta \mathrm{n}-\theta \mathrm{d})
$$

where, $\mathrm{Fd}$ is the fraction of denatured nucleic acid, $\theta \mathrm{n}$ the minimum ellipticity at $210 \mathrm{~nm}$ or the maximum ellipticity at $260 \mathrm{~nm}, \theta \mathrm{d}$ the maximum ellipticity at $210 \mathrm{~nm}$ or the minimum ellipticity at $260 \mathrm{~nm}$, and $\theta$ obs the observed ellipticity at Fd. The ellipticity, $\theta$ obs, when $\mathrm{Fd}=0.5$ was calculated and used to calculate $\mathrm{Tm}$ from the linear fit of the transition region. For comparisons between different RNA samples, the first derivative of the ellipticity, $\theta$ obs, the change in molar ellipticity as a function of temperature $(\mathrm{d} \theta / \mathrm{d} T)$, was calculated and plotted.

Statistical analysis. All statistical analyses were performed using GraphPad Prism 7.0. For comparison of PAR-CLIP and RNA-seq data sets, we used multiple unpaired $t$ tests with the Benjamini-Hochberg correction to identify areas in which these sequencing preparations were statistically different from each other $(Q<0.1$ and $Q<0.01)$. Transition-distance was determined using FDR $(Q<0.05)$. For analysis of the focus-forming assay, luciferase assay, and lung viral titers, we used one-way ANOVA with multiple comparison corrections (Kruskal-Wallis test). TCID $_{50}$ per HA-unit was analyzed using an unpaired $t$ test.

Data availability. The unprocessed PAR-CLIP and RNA-seq files have been uploaded to the NCBI Sequence Read Archive (SRA, SUB3042681). All other data supporting the findings of this study are available within the article and its Supplementary Information files, or are available from the authors upon request.

Received: 22 May 2017 Accepted: 4 January 2018

Published online: 31 January 2018

\section{References}

1. Arranz, R. et al. The structure of native influenza virion ribonucleoproteins. Science 338, 1634-1637 (2012).

2. Moeller, A., Kirchdoerfer, R. N., Potter, C. S., Carragher, B. \& Wilson, Ia Organization of the influenza virus replication machinery. Science 338 , 1631-1634 (2012).

3. Stockley, P. G. et al. Packaging signals in single-stranded RNA viruses: Nature's alternative to a purely electrostatic assembly mechanism. J. Biol. Phys. 39, 277-287 (2013)

4. Nicholson, B. L. \& White, K. A. Functional long-range RNA-RNA interactions in positive-strand RNA viruses. Nat. Rev. Microbiol. 12, 493-504 (2014)

5. Gultyaev, A. P. et al. RNA structural constraints in the evolution of the influenza A virus genome NP segment. Rna. Biol. 11, 942-952 (2014).

6. Moss, W. N., Priore, S. F. \& Turner, D. H. Identification of potential conserved RNA secondary structure throughout influenza A coding regions. RNA 17, 991-1011 (2011).

7. Kobayashi, Y. et al. Computational and molecular analysis of conserved influenza A virus RNA secondary structures involved in infectious virion production. Rna. Biol. 13, 883-894 (2016).

8. York, A., Hengrung, N., Vreede, F. T., Huiskonen, J. T. \& Fodor, E. Isolation and characterization of the positive-sense replicative intermediate of a negative-strand RNA virus. Proc. Natl Acad. Sci. USA 110, E4238-E4245 (2013).

9. Klumpp, K., Ruigrok, R. W. H. \& Baudin, F. Roles of the influenza virus polymerase and nucleoprotein in forming a functional RNP structure. EMBO J. 16, 1248-1257 (1997).

10. Goto, H., Muramoto, Y., Noda, T. \& Kawaoka, Y. The genome-packaging signal of the influenza A virus genome comprises a genome incorporation signal and a genome-bundling signal. J. Virol. 87, 11316-11322 (2013).

11. Marsh, Ga, Rabadán, R., Levine, A. J. \& Palese, P. Highly conserved regions of influenza a virus polymerase gene segments are critical for efficient viral RNA packaging. J. Virol. 82, 2295-2304 (2008).

12. Fournier, E. et al. Interaction network linking the human $\mathrm{H} 3 \mathrm{~N} 2$ influenza $A$ virus genomic RNA segments. Vaccine 30, 7359-7367 (2012).

13. Essere, B. et al. Critical role of segment-specific packaging signals in genetic reassortment of influenza A viruses. Proc. Natl Acad. Sci. USA 110, E3840-E3848 (2013).

14. Fournier, E. et al. A supramolecular assembly formed by influenza A virus genomic RNA segments. Nucleic Acids Res. 40, 2197-2209 (2011).

15. Ozawa, M. et al. Nucleotide sequence requirements at the 5' end of the influenza A virus M RNA segment for efficient virus replication. J. Virol. 83, 3384-3388 (2009).

16. Fournier, E. et al. A supramolecular assembly formed by influenza A virus genomic RNA segments. Nucleic Acids Res. 40, 2197-2209 (2012).

17. Noda, T. et al. Three-dimensional analysis of ribonucleoprotein complexes in influenza A virus. Nat. Commun. 3, 639 (2012).

18. Nakatsu, S. Complete and incomplete genome packaging of influenza A and B viruses. MBio 7, e01248-16 (2016).

19. Lakdawala, S. S. et al. Influenza a virus assembly intermediates fuse in the cytoplasm. PLoS Pathog. 10, e1003971 (2014).

20. Gerber, M., Isel, C., Moules, V. \& Marquet, R. Selective packaging of the influenza A genome and consequences for genetic reassortment. Trends Microbiol. 22, 446-455 (2014)

21. Kutluay, S. B. et al. Global changes in the RNA binding specificity of HIV-1 gag regulate virion genesis. Cell 159, 1096-1109 (2014).

22. Marsh, Ga., Hatami, R. \& Palese, P. Specific residues of the influenza A virus hemagglutinin viral RNA are important for efficient packaging into budding virions. J. Virol. 81, 9727-9736 (2007).

23. Brooke, C. B. et al. Most influenza A virions fail to express at least one essential viral protein. J. Virol. 87, 3155-3162 (2013).

24. c. Nick, P. Measuring and increasing protein stability. Tibtech 8, 93-98 (1990).

25. Liu, C. L. et al. Using mutagenesis to explore conserved residues in the RNAbinding groove of influenza A virus nucleoprotein for antiviral drug development. Sci. Rep. 6, 21662 (2016).

26. Zheng, W., Olson, J., Vakharia, V. \& Tao, Y. J. The crystal structure and RNAbinding of an orthomyxovirus nucleoprotein. PLoS Pathog. 9, e1003624 (2013).

27. Lee, N. et al. Genome-wide analysis of influenza viral RNA and nucleoprotein association. Nucleic Acids Res. 5, e1000491 (2017).

28. Gallagher, J. R., Torian, U., McCraw, D. M. \& Harris, A. K. Structural studies of influenza virus RNPs by electron microscopy indicate molecular contortions within NP supra-structures. J. Struct. Biol. 197, 294-307 (2016).

29. Dou, D. et al. Analysis of IAV replication and co-infection dynamics by a versatile RNA viral genome labeling method. Cell Rep. 20, 251-263 (2017).

30. Hutchinson, E. C., Wise, H. M., Kudryavtseva, K., Curran, M. D. \& Digard, P. Characterisation of influenza A viruses with mutations in segment 5 packaging signals. Vaccine 27, 6270-6275 (2009). 
31. Gao, Q. et al. The influenza A virus PB2, PA, NP, and M segments play a pivotal role during genome packaging. J. Virol. 86, 7043-7051 (2012).

32. Yamanaka, K., Ishihama, A. \& Nagata, K. Reconstitution of influenza virus RNA-nucleoprotein complexes structurally resembling native viral ribonucleoprotein cores. J. Biol. Chem. 265, 11151-11155 (1990).

33. Gilbertson, B. et al. Influenza NA and PB1 gene segments interact during the formation of viral progeny: localization of the binding region within the PB1 gene. Viruses 8, 1-17 (2016).

34. Cobbin, J. Ca et al. Influenza virus PB1 and NA gene segments can cosegregate during vaccine reassortment driven by interactions in the PB1 coding region. J. Virol. 88, 8971-8980 (2014)

35. Williams, G. D., Pinto, A. K., Doll, B. \& Boon, A. C. M. A North American H7N3 influenza virus supports reassortment with 2009 pandemic H1N1 and induces disease in mice without prior adaptation. J. Virol. 90, JVI.02761-15 (2016).

36. Gokhale, N. S. et al. N6-methyladenosine in flaviviridae viral RNA genomes regulates infection. Cell. Host Microbe 20, 654-665 (2016).

37. Gruber, A. R., Lorenz, R., Bernhart, S. H., Neuböck, R. \& Hofacker, I. L. The vienna RNA websuite. Nucleic Acids Res. 36, W70-W74 (2008).

38. Dawson, W. K., Fujiwara, K. \& Kawai, G. Prediction of RNA pseudoknots using heuristic modeling with mapping and sequential folding. PLOS ONE $\mathbf{2}$, e905 (2007).

39. Schindelin, J. et al. Fiji: an open-source platform for biological-image analysis. Nat. Methods 9, 676-682 (2012).

40. Reed, L. J. \& Muench, H. A simple method of estimating fifty per cent endpoints. Am. J. Hyg. 27, 493-497 (1938).

41. Hyde, J. L. et al. A viral RNA structural element alters host recognition of nonself RNA. Science 343, 783-788 (2014).

\section{Acknowledgements}

We thank the following colleagues for comments on experiments comprising this manuscript: Michael Diamond, MD, David Wang, PhD, Daisy W. Leung, PhD, and Deborah Lenschow, MD and Anshu Gounder all of Washington University in Saint Louis. Additionally, Traci Bricker and Christopher Edwards provided technical support. Chris Brooke provided anti-M1 hybridoma, M2-1C6. NIGMS training grant 5T32GM007067 and NIAID ID Training Grant 2T32AI007172, and the Victoria Fraser Infectious Disease Research Fellowship provided funding to GW. This study was funded in part by the National Institute of Health (NIH) grants P01AI120943 (G.K.A) and R01AI118938 (A.C.M.B.)

\section{Author contributions}

G.D.W. and A.C.M.B. conceived and performed the experiments, analyzed the data, and wrote the manuscript. S.B.K. and D.T. provided technical and conceptual assistance in performing PAR-CLIP experiments. K.M.W. provided bioinformatics analysis support. G.K.A. assisted with the analysis and conception of certain experiments concerning RNA structure. P.J.K. and G.K.A. performed the RNA thermostability assays.

\section{Additional information}

Supplementary Information accompanies this paper at https://doi.org/10.1038/s41467018-02886-w.

Competing interests: The authors declare no competing financial interests.

Reprints and permission information is available online at http://npg.nature.com/ reprintsandpermissions/

Publisher's note: Springer Nature remains neutral with regard to jurisdictional claims in published maps and institutional affiliations.

\begin{abstract}
(c) (i) Open Access This article is licensed under a Creative Commons Attribution 4.0 International License, which permits use, sharing, adaptation, distribution and reproduction in any medium or format, as long as you give appropriate credit to the original author(s) and the source, provide a link to the Creative Commons license, and indicate if changes were made. The images or other third party material in this article are included in the article's Creative Commons license, unless indicated otherwise in a credit line to the material. If material is not included in the article's Creative Commons license and your intended use is not permitted by statutory regulation or exceeds the permitted use, you will need to obtain permission directly from the copyright holder. To view a copy of this license, visit http://creativecommons.org/ licenses/by/4.0/.
\end{abstract}

(C) The Author(s) 2018 